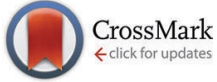

Cite this: Phys. Chem. Chem. Phys., 2015, 17, 20093

Received 28th April 2015, Accepted 5th June 2015

DOI: $10.1039 / c 5 c p 02461 g$

www.rsc.org/pccp

\section{Computational simulation and interpretation of the low-lying excited electronic states and electronic spectrum of thioanisole $\uparrow$}

\begin{abstract}
Shaohong L. Li, Xuefei Xu and Donald G. Truhlar*
Three singlet states, namely a closed-shell ground state and two excited states with ${ }^{1} \pi \pi^{*}$ and ${ }^{1}$ n $\sigma^{*}$ character, have been suggested to be responsible for the radiationless decay or photochemical reaction of photoexcited thioanisole. The correct interpretation of the electronic spectrum is critical for understanding the character of these low-lying excited states, but the experimental spectrum is yet to be fully interpreted. In the work reported here, we investigated the nature of those three states and a fourth singlet state of thioanisole using electronic structure calculations by multireference perturbation theory, by completelyrenormalized equation-of-motion coupled cluster theory with single and double excitations and noniterative inclusion of connected triples (CR-EOM-CCSD(T)), and by linear-response time-dependent density functional theory (TDDFT). We clarified the assignment of the electronic spectrum by simulating it using a normal-mode sampling approach combined with TDDFT in the Tamm-Dancoff approximation (TDA). The understanding of the electronic states and of the accuracy of the electronic structure methods lays the foundation of our future work of constructing potential energy surfaces.
\end{abstract}

\section{Introduction}

As discussed by Sobolewski, Domcke, and coworkers, ${ }^{1-4}$ many organic molecules with aromatic rings and heteroatoms have photoinduced hydrogen detachment channels in the gas phase and proton transfer channels in protic solvents that are mediated by a repulsive ${ }^{1} \pi \sigma^{*}$ or ${ }^{1} n \sigma^{*}$ state. Among these, phenol and thiophenol, as well as their derivatives anisole and thioanisole, exhibit qualitatively similar potential energy profiles that may be considered prototypes of this behavior. ${ }^{5-13}$ For thioanisole, the existence and nature of the ${ }^{1} n \sigma^{*}$ state has been identified and discussed in previous work. ${ }^{13,14}$ It was conjectured, based on complete active space self-consistent field (CASSCF) calculations and spectroscopic studies, that the dynamics of the photodissociation of thioanisole into thiophenoxyl and methyl radicals is facilitated by the coupling of the optically accessible ${ }^{1} \pi \pi^{*}$ state to the dark ${ }^{1} n \sigma^{*}$ state. The detailed mechanism of the reaction is, however, still open to question.

Our ultimate goal is to use theory and computation to gain more insight into the mechanism, by constructing the potential energy surfaces of the relevant ground and excited states and

Department of Chemistry, Chemical Theory Center, and Supercomputing Institute, University of Minnesota, Minneapolis, Minnesota 55455, USA.

E-mail: truhlar@umn.edu

$\dagger$ Electronic supplementary information (ESI) available. See DOI: 10.1039/ c5cp02461g performing dynamical simulation on the coupled surfaces. As the first step, in this work we study the ultraviolet absorption spectrum of thioanisole to obtain more information about the character of the electronic excited states in the Franck-Condon region and to validate electronic structure methods for calculating these states. A vapor-phase experimental spectrum was reported in a recent article ${ }^{14}$ but has yet to be fully interpreted and understood. Instead of assigning states to the spectral bands simply according to their vertical excitation energies and transition probabilities, we attempt to simulate the spectrum using a normal-mode sampling strategy as detailed below so as to directly compare the simulated profile to the experimental spectrum.

The rest of this paper will proceed as follows. After describing the computational details in Section 2, we discuss in Section 3 the optimized geometries of $S_{0}$ and $S_{1}$, the character of the excited states, and the interpretation of the electronic spectrum based on our simulation. Finally we conclude our discussion in Section 4.

\section{Computational details}

2.1 Basis sets, electronic structure methods, and software

We used five basis sets. In order of decreasing completeness, they are: 
- augmented correlation-consistent polarized valence triple zeta with an additional tight $\mathrm{d}$ function for sulfur: aug-cc-pV(T+d)Z; ${ }^{15-17}$

- minimally augmented, multiply polarized valence triple zeta: MG3S; ${ }^{18}$

- minimally augmented, polarized valence triple zeta (multiply polarized for sulfur), denoted as MB hereafter: $6-311+G(d)^{19,20}$ for carbon and hydrogen and MG3S for sulfur;

- partially augmented, correlation-consistent polarized valence double zeta with an additional spherical harmonic set of tight d functions for sulfur: jun-cc-pV(D+d)Z; $;^{15-17,21}$

- minimally augmented, polarized valence double zeta: $6-31+\mathrm{G}(\mathrm{d}) ;^{20,22-24}$

We used both Kohn-Sham density functional theory (KS-DFT) and wave function theory (WFT) for electronic structure calculations. The levels of theory and their abbreviations are listed in Table 1 along with ref. 25-42 that explain each level of theory.

We used the following software to perform various types of calculations: Gaussian 09, ${ }^{43}$ GAMESS ${ }^{44,45}$ NWChem ${ }^{46}$ ANT (incorporating the MOPAC $5.021 \mathrm{mn}$ code), ${ }^{47,48}$ and Multiwfn. ${ }^{49}$ The calculations done with each software package are listed in Table 2.

\subsection{Coordinates and geometry optimization}

The equilibrium geometry of both $\mathrm{S}_{0}$ and $\mathrm{S}_{1}$ has $C_{\mathrm{s}}$ symmetry with the symmetry plane in the phenyl ring. The equilibrium geometry of $S_{0}$ is shown in Fig. 1, which also illustrates our convention for numbering the carbon atoms. The molecular orientation is defined by putting the $\mathrm{C} 2, \mathrm{C} 1$, and $\mathrm{S}$ atoms in the $x y$ plane with the $\mathrm{C} 1-\mathrm{S}$ bond pointing to the positive $x$ direction.

The ground-state equilibrium geometry was optimized by M06-2X/MG3S and by CASSCF(12,11)/MB. ${ }^{50}$ The equilibrium geometry of $\mathrm{S}_{1}$ was also optimized by $\operatorname{CASSCF}(12,11) / \mathrm{MB}$. The 11 active orbitals used in the $\operatorname{CASSCF}(12,11)$ calculations
Table 2 Software and calculations

\begin{tabular}{lll}
\hline Software & $\begin{array}{l}\text { Ref. for } \\
\text { software }\end{array}$ & Calculations done with the software \\
\hline Gaussian 09 & 43 & KS-DFT, TDDFT, TDA-TDDFT \\
GAMESS & 44,45 & $\begin{array}{l}\text { CASSCF, SA-CASSCF, MC-QDPT, XMC-QDPT } \\
\text { NWChem }\end{array}$ \\
ANT & 46 & $\begin{array}{l}\text { EOM-CCSD, CR-EOM-CCSD(T) } \\
\text { Geometry sampling for the simulation of } \\
\text { electronic spectrum } \\
\text { Gaussian broadening for the simulation of } \\
\text { electronic spectrum }\end{array}$ \\
Multiwfn & 49 &
\end{tabular}

nominally correspond to three $\pi$ and three $\pi^{*}$ on the phenyl ring, two $\sigma_{\mathrm{C}-\mathrm{S}}$ and two $\sigma_{\mathrm{C}-\mathrm{s}^{*}}$ on the C1-S and S-C7 bonds, and $\mathrm{n}\left(p_{z}\right)$ on the sulfur (see Fig. 2). All the active orbitals delocalize to some extent; for example, the two $\sigma_{\mathrm{C}-\mathrm{S}}$ have components on both the $\mathrm{C} 1-\mathrm{S}$ and $\mathrm{S}-\mathrm{C} 7$ bonds, and the orbital we label as $\mathrm{n}\left(p_{z}\right)$ has both a component on $\mathrm{S}$ and a $\pi$ component on the ring.

\subsection{Excitation energies and simulation of the electronic absorption spectrum in the vapor phase}

The vertical excitation energies (VEEs) to the two or three lowest singlet excited states in the Franck-Condon region were calculated by MC-QDPT, XMC-QDPT, EOM-CCSD, CR-EOM-CCSD(T), TDDFT, and TDA-TDDFT. (X)MC-QDPT calculations were carried out with a three-dimensional model space based on an $\operatorname{SA}(3)-\operatorname{CASSCF}(12,11)$ reference. The 11-orbital active space has the same character as described in Section 2.2 .

Our strategy to simulate the spectrum of the vapor-phase thioanisole is to sample a certain number of representative geometries according to the ground-state vibrational distribution, calculate their contributions to the spectrum, and add the contributions. In particular, we first optimized the ground-state geometry of thioanisole and performed normal mode analysis

Table 1 Electronic structure methods

\begin{tabular}{|c|c|c|c|}
\hline Type & Abbreviation & Name of method & Ref. \\
\hline & TD-M06-2X & Linear-response time-dependent density functional theory (TDDFT) with the & 26 \\
\hline & & M06-2X exchange-correlation functional & 25 \\
\hline & TDA- $\tau$-HCTHhyb & $\begin{array}{l}\text { TDA-TDDFT (TDDFT with the Tamm-Dancoff approximation) with the } \\
\tau \text {-HCTHhyb exchange-correlation functional }\end{array}$ & $28-31$ \\
\hline \multirow[t]{6}{*}{ WFT } & $\operatorname{CASSCF}(n, m)$ & $\begin{array}{l}\text { Single-state complete active space self-consistent-field theory with } n \text { active } \\
\text { electrons in } m \text { active orbitals }\end{array}$ & 32 \\
\hline & MC-QDPT $^{c}$ & Multi-configurational quasi-degenerate perturbation theory & 34,35 \\
\hline & $\mathrm{XMC}-\mathrm{QDPT}^{c}$ & Extended multi-configurational quasi-degenerate perturbation theory & 36 \\
\hline & $\mathrm{EOM}-\mathrm{CCSD}^{d}$ & Equation-of-motion coupled cluster theory with singles and doubles & 37,38 \\
\hline & CR-EOM-CCSD $(\mathrm{T})^{d, e}$ & $\begin{array}{l}\text { Completely-renormalized equation-of-motion coupled cluster theory with singles, } \\
\text { doubles, and noniterative connected triples }\end{array}$ & 39,40 \\
\hline & PM3 & Parametrized model 3 & 41,42 \\
\hline
\end{tabular}

${ }^{a}$ All KS-DFT calculations in this paper used an ultrafine (99590) grid with 99 radial shells and 590 grid points per shell for numerical integration except the TDA-TDDFT calculations for simulating the electronic spectrum, which used a fine $(75302)$ grid. ${ }^{b}$ SA $(N)$-CASSCF denotes that the average is over $N$ states. In this article, state averages are always carried out with equal weights for all states averaged. ${ }^{c}$ All MC-QDPT and XMCQDPT calculations were carried out with intruder state avoidance ${ }^{56}$ with an energy denominator shift of $0.02 E_{\mathrm{h}} \cdot{ }^{d}$ In these calculations, the $1 \mathrm{~s}$ orbitals on $\mathrm{H}$ and $\mathrm{C}$ and the $1 \mathrm{~s}, 2 \mathrm{~s}$, and 2 p orbitals on $S$ were not correlated. ${ }^{e}$ All CR-EOM-CCSD(T) calculations were carried out with variant IA of the method for the so-called $\delta$-corrected excitation energies which incorporates a noniterative connected triples correction to EOM-CCSD excitation energies rather than to state energies. 


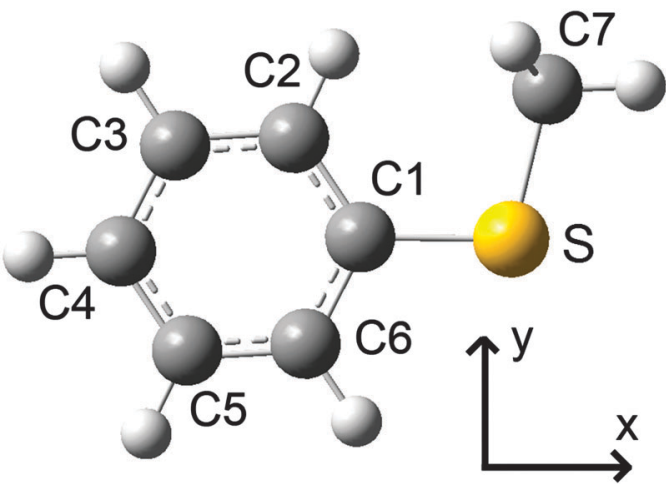

Fig. 1 Equilibrium geometry of thioanisole with $C_{\mathrm{s}}$ symmetry as optimized by M06-2X/MG3S. Orientation of the $x$ and $y$ axes and numbering of the carbon atoms for convenience of description are also shown.

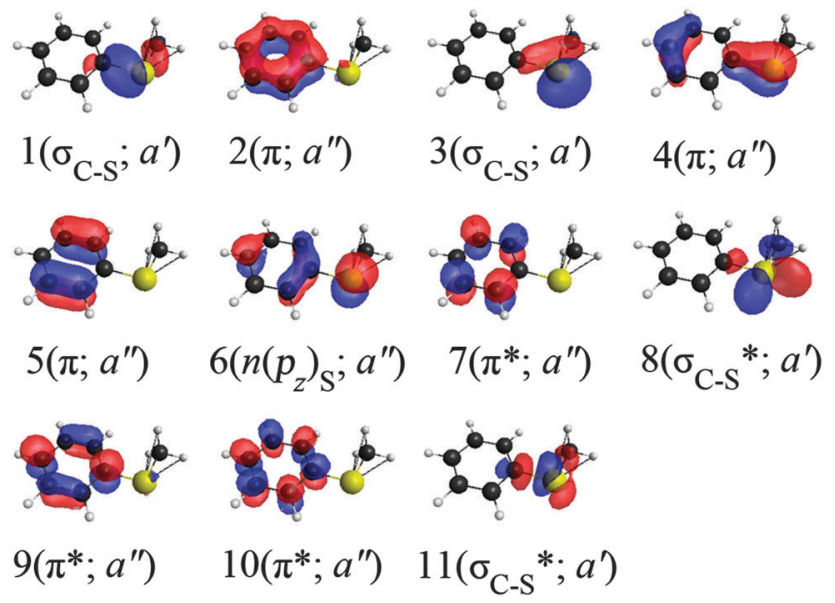

Fig. 2 Schematic of three-state-averaged active orbitals at the equilibrium geometry with $C_{s}$ symmetry from SA-CASSCF calculations. The character and the irrep of the orbitals are given in parentheses.

by the semiempirical PM3 method. We then sampled 200 geometries with a ground-state harmonic oscillator distribution along each Cartesian normal coordinate. For each sampled geometry, we calculated the VEEs and oscillator strengths of the four lowest excited states using TDA- $\tau-\mathrm{HCTHhyb} / 6-31+\mathrm{G}(\mathrm{d})$. These VEEs and oscillator strengths from the sampled geometries were collected to generate a stick spectrum. This stick spectrum accounts for the broadening and peak shift of the spectrum due to the statistical distribution of geometries generated by ground-state molecular vibrations, taking into account also the dependence of transition probabilities on geometry. Finally, we broadened the sticks with Gaussian functions with half width at half maximum (HWHM) equal to $0.15 \mathrm{eV}$ to produce a smooth profile of electronic spectrum. The Gaussian broadening mimics the missing effects such as broadening due to lifetime broadening, thermally excited vibrations, rotational broadening, anharmonic broadening, and instrumental resolution. At the longest wavelengths, the resolution is presumably limited by spectral congestion (the jet-cooled excitation spectrum in ref. 51 shows resolved rovibrational structure). At shorter wavelengths, lifetime broadening probably dominates.

\section{Results and discussion}

\subsection{Optimized geometries of $S_{0}$ and $S_{1}$}

The optimized geometry of $\mathrm{S}_{0}$ has $C_{\mathrm{s}}$ symmetry with phenyl, $\mathrm{S}$, $\mathrm{C} 7$, and one $\mathrm{H}$ of the methyl group in a plane and the other two hydrogens of the methyl group above and below the plane. (See the ESI $\dagger$ for the coordinates and vibrational frequencies of the optimized geometries.) The length of the $\mathrm{C} 1-\mathrm{S}$ and $\mathrm{S}-\mathrm{C} 7$ bonds given by M06-2X (by CASSCF) are $1.76 \AA$ (1.80 ̊) and $1.80 \AA$ $(1.83 \AA)$ respectively. The C1-S-C7 bond angle given by M06-2X (by CASSCF) is $102.88^{\circ}\left(103.84^{\circ}\right)$. The length of the $\mathrm{C}-\mathrm{C}$ bonds in the phenyl ring is in the range of $1.38-1.40 \AA$ by either method. Originally there was debate on whether the $\mathrm{C} 2-\mathrm{C} 1-\mathrm{S}-\mathrm{C} 7$ torsion at the ground-state equilibrium of thioanisole is zero or not, but recent experiments and electronic structure calculations support the former, ${ }^{51,52}$ with which our results are consistent. Since M06-2X is more reliable for geometries than CASSCF (because the latter lacks dynamic correlation), we use the M06-2X geometry as the equilibrium geometry for $S_{0}$ in the following calculations, except for the calculation of adiabatic excitation energy and the simulation of spectrum as will be discussed below.

The optimized geometry of $\mathrm{S}_{1}$ by $\operatorname{CASSCF}(12,11) / \mathrm{MB}$ is similar to the $\mathrm{S}_{0}$ equilibrium geometry optimized at the same level, with the $\mathrm{C}-\mathrm{C}$ bonds in the phenyl ring lengthened by $\sim 0.03 \AA$, the C1-S bond shortened by $0.03 \AA$, the S-C7 bond lengthened by $0.02 \AA$, and the $\mathrm{C} 1-\mathrm{S}-\mathrm{C} 7$ bond angle increased by $1^{\circ}$, which is also consistent with previous work. ${ }^{51}$

\subsection{Nature of the four lowest singlet states and electronic spectroscopy}

For the photochemistry of thioanisole with the low excitation energies $(\sim 4.2-4.5 \mathrm{eV})$ used in the experiments, ${ }^{13,14}$ only the two lowest singlet electronically excited states $\left(S_{1}\right.$ and $\left.S_{2}\right)$ are deemed to be important. However, the third excited singlet state $\left(\mathrm{S}_{3}\right)$ makes an important contribution to the spectrum, so in this section we consider the four lowest singlet states. At the equilibrium geometry of the ground state with $C_{\mathrm{s}}$ symmetry, $\mathrm{S}_{0}$ is a closed-shell state belonging to the $\mathrm{A}^{\prime}$ irreducible representation (irrep), $S_{1}$ and $S_{3}$ are ${ }^{1} \pi \pi^{*}$ states (simply to be called $\pi \pi^{*}$ ) belonging to the $\mathrm{A}^{\prime}$ irrep, and $\mathrm{S}_{2}$ is a ${ }^{1} n \sigma^{*}$ state (simply to be called $n \sigma^{*}$ ) belonging to the $\mathrm{A}^{\prime \prime}$ irrep. The dominant configurations of the four states given by SA-CASSCF are listed in Table 3. Table 3 actually shows that one of the dominant configurations of both $S_{1}$ and $S_{3}$ has $n \pi^{*}$ character, but considering the facts that the other dominant configuration is $\pi \pi^{*}$ and that the $n$ orbital also has $\pi$ character, we simply follow other authors ${ }^{13,14}$ and label $S_{1}$ and $S_{3}$ as $\pi \pi^{*}$.

The vertical excitation energies (VEEs) calculated by a variety of electronic structure methods are listed in Table 4. There are several messages we can take from this table. Firstly, MC-QDPT and XMC-QDPT based on the same SA-CASSCF reference give essentially the same VEEs at the $\mathrm{S}_{0}$ equilibrium geometry. Secondly, SA(3)-MC-QDPT(12,11) and CR-EOM-CCSD(T), which are very different kinds of high-level methods, give very similar VEEs when used with the extensive aug-cc-pV $(\mathrm{T}+\mathrm{d}) \mathrm{Z}$ basis set, which suggests that both should be quite accurate. 
Table 3 The $\mathrm{Cl}$ coefficients of the dominant configurations of the four lowest singlet states of thioanisole at the equilibrium geometry ${ }^{a}$ as calculated by SA(4)-CASSCF(12,11)/MB

\begin{tabular}{lll}
\hline State & Dominant configuration $(\mathrm{s})^{b}$ & CI coefficient \\
\hline $\mathrm{S}_{0}$ & 22222200000 & 0.93 \\
$\mathrm{~S}_{1}$ & 22222110000 & 0.62 \\
& 22221200100 & 0.58 \\
$\mathrm{~S}_{2}$ & 22222101000 & 0.84 \\
& 22212201000 & 0.33 \\
$\mathrm{~S}_{3}$ & 22222100100 & 0.86 \\
& 22212200100 & 0.26
\end{tabular}

${ }^{a}$ Geometry optimized by M06-2X/MG3S. ${ }^{b}$ The occupation numbers of the dominant configurations correspond to orbitals in Fig. 2 in the same order.

Thirdly, TDA- $\tau$-HCTHhyb with a small $6-31+G^{*}$ basis set gives VEEs close to our best estimate values from CR-EOM-CCSD(T)/ aug-cc-pV $(\mathrm{T}+\mathrm{d}) \mathrm{Z}$. Because of the accuracy and low computational cost of TDA- $\tau-H C T H h y b / 6-31+G^{*}$, we use it for the simulation of electronic spectrum discussed later.

To further validate the methods we use, we calculate the $\mathrm{S}_{1} \leftarrow \mathrm{S}_{0}$ adiabatic excitation energy by using the following formula:

$$
\Delta E^{(0-0)}\left(\mathrm{S}_{1} \leftarrow \mathrm{S}_{0}\right)=V_{\mathrm{e}}\left(\mathrm{S}_{1}\right)+\mathrm{ZPE}\left(\mathrm{S}_{1}\right)-V_{\mathrm{e}}\left(\mathrm{S}_{0}\right)-\mathrm{ZPE}\left(\mathrm{S}_{0}\right)
$$

where $V_{\mathrm{e}}\left(\mathrm{S}_{i}\right)(i=0,1)$ is the electronic energy of $\mathrm{S}_{i}$ calculated by SA(3)-MC-QDPT(12,11)/MB at the $\mathrm{S}_{i}$ geometry optimized by $\operatorname{CASSCF}(12,11) / \mathrm{MB}$, and $\mathrm{ZPE}\left(\mathrm{S}_{i}\right)$ is the zero-point energy of $\mathrm{S}_{i}$ calculated by $\operatorname{CASSCF}(12,11) / \mathrm{MB}$. The calculated adiabatic excitation energy is $4.22 \mathrm{eV}$, in excellent agreement with the experimental value of $4.28 \mathrm{eV}^{13,14,51}$

The $\mathrm{S}_{1}$ state is often called a "bright" state ${ }^{14}$ even though this description makes sense as compared to the dark $n \sigma^{*}$ state, some readers may think it corresponds to the bright band of the experimental vapor-phase UV absorption spectrum which peaks at $4.96 \mathrm{eV}$ [see Fig. 3(a)]. This view may be strengthened by our EOM-CCSD/aug-cc-pV $(\mathrm{T}+\mathrm{d}) \mathrm{Z}$ calculation, which gives the $S_{1} \leftarrow S_{0}$ VEE equal to $4.84 \mathrm{eV}$ (see Table 4). However, if we compare our best estimate of the $\mathrm{S}_{1} \leftarrow \mathrm{S}_{0}$ VEE by CR-EOM-CCSD(T) $(4.53 \mathrm{eV})$ to the maximum of the experimental

Table 4 Vertical excitation energies (in eV) as calculated by different methods and basis sets ${ }^{a}$

\begin{tabular}{lllll}
\hline Method & Basis set & $\mathrm{S}_{1}\left(2 \mathrm{~A}^{\prime}\right)$ & $\mathrm{S}_{2}\left(1 \mathrm{~A}^{\prime \prime}\right)$ & $\mathrm{S}_{3}\left(3 \mathrm{~A}^{\prime}\right)$ \\
\hline EOM-CCSD & aug-cc-pV(T+d)Z & 4.84 & 5.21 & 5.52 \\
CR-EOM-CCSD(T) & aug-cc-pV(T+d)Z & 4.53 & 5.03 & 5.25 \\
TD-M06-2X & MG3S & 4.94 & 5.05 & 5.30 \\
TD-B3LYP & MB & 4.60 & 4.82 & 5.00 \\
TDA- $\tau$-HCTHhyb & 6-31+G* & 4.65 & 4.97 & 5.18 \\
TD-CAM-B3LYP & aug-cc-pVTZ & 4.95 & 5.14 & 5.31 \\
SA(3)-MC-QDPT(12,11) & MB & 4.64 & 5.13 & - \\
SA(3)-XMC-QDPT(12,11) & MB & 4.64 & 5.13 & - \\
SA(3)-MC-QDPT(12,11) & aug-cc-pV(T+d)Z & 4.52 & 5.02 & -
\end{tabular}

${ }^{a}$ All data are from this work, calculated at the equilibrium geometry optimized by M06-2X/MG3S, unless specified otherwise. ${ }^{b}$ Ref. 14 .
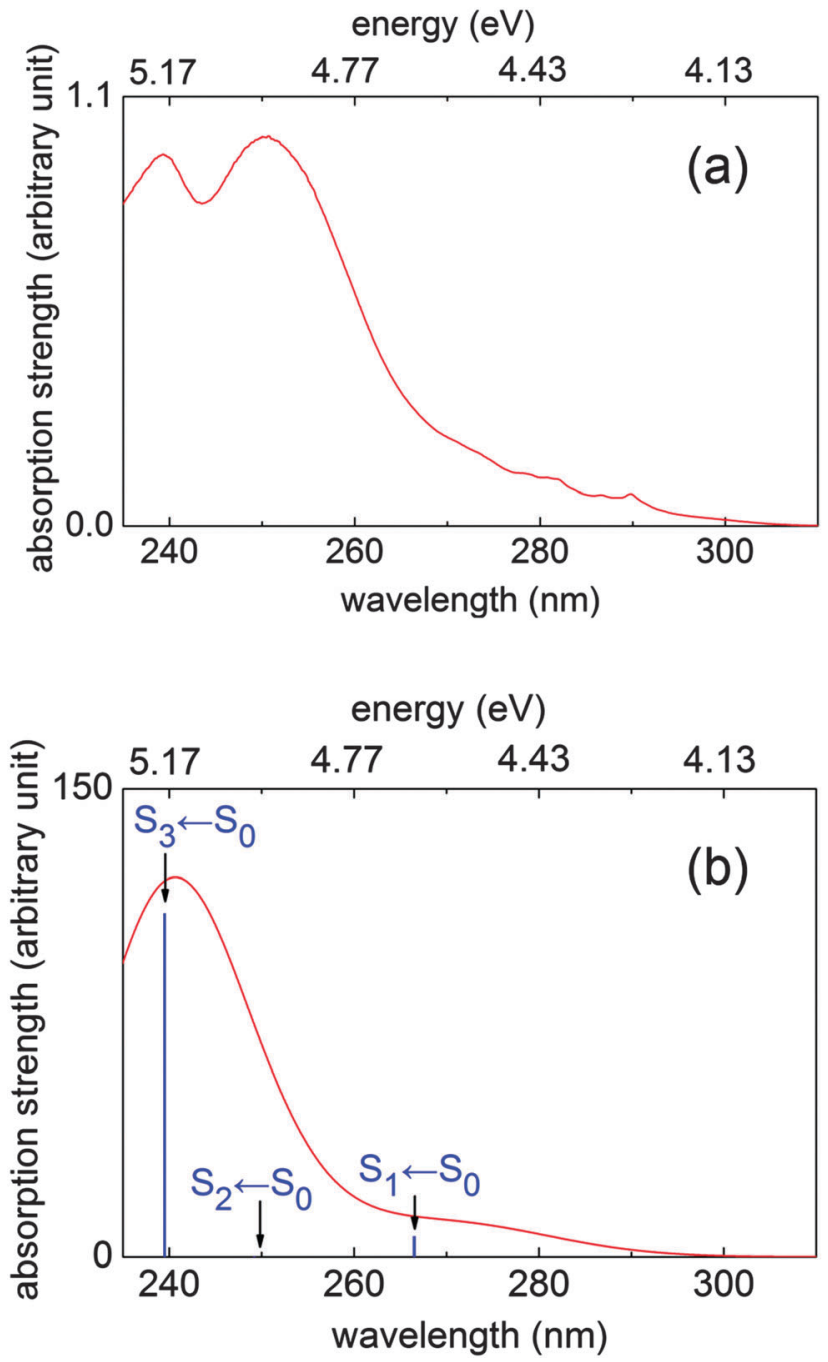

Fig. 3 (a) Experimental vapor-phase UV spectrum of thioanisole. (Adapted from Fig. S6 in the Electronic Supplementary Information of ref. 14 with the permission of the authors.) (b) Simulated electronic spectrum of thioanisole (red curve) and the position of vertical excitation energies (blue sticks) as calculated by TDA- $\tau-$ HCTHhyb/6-31+G*

spectrum (4.96 eV), the discrepancy is as large as $0.4 \mathrm{eV}$. On the other hand, the experimental adiabatic excitation energy of $4.28 \mathrm{eV}^{13,14}$ also differs significantly from the maximum of the spectral band. These facts imply that the experimentally bright band at $4.96 \mathrm{eV}$ may not correspond to the $\mathrm{S}_{1} \leftarrow \mathrm{S}_{0}$ excitation.

There have been disagreements in the literature about the nature of states and the interpretation of the electronic spectrum. For instance, Lim et al. ${ }^{13}$ and Roberts et al. ${ }^{14}$ reported $\mathrm{S}_{1}$ as a bright $\pi \pi^{*}$ state and $\mathrm{S}_{2}$ a dark $n \sigma^{*}$ state, and Roberts et al. assigned the strong absorption band peaked at $4.96 \mathrm{eV}$ to $\mathrm{S}_{3} \leftarrow \mathrm{S}_{0}$ transition based on their single-point TD-CAM-B3LYP, LR-CCSD, and CCSDR(3) calculations, although their calculated $\mathrm{S}_{3} \leftarrow \mathrm{S}_{0}$ VEE is higher than $5.3 \mathrm{eV}$. Liu et al. ${ }^{53}$ on the other hand, reported $S_{1}$ as a dark $\pi \pi^{*}$ state, $S_{2}$ a bright $\pi \pi^{*}$ state, and $\mathrm{S}_{3}$ a dark $n \sigma^{*}$ state based on their TD-B3LYP/6-31G(d) calculation, assigning the experimental band to the $\mathrm{S}_{2} \leftarrow \mathrm{S}_{0}$ transition. Our calculations of VEEs (Table 4) and oscillator strengths 
Table 5 Oscillator strengths as calculated by different methods and basis sets $^{a}$

\begin{tabular}{lllll}
\hline Method & Basis set & $\mathrm{S}_{1}\left(2 \mathrm{~A}^{\prime}\right)$ & $\mathrm{S}_{2}\left(1 \mathrm{~A}^{\prime \prime}\right)$ & $\mathrm{S}_{3}\left(3 \mathrm{~A}^{\prime}\right)$ \\
\hline EOM-CCSD & jun-cc-pV(D+d)Z & 0.0086 & 0.0000 & 0.2745 \\
TD-M06-2X & MG3S & 0.0177 & 0.0001 & 0.2628 \\
TD-B3LYP & MB & 0.0158 & 0.0001 & 0.2433 \\
TDA- $\tau$-HCTHhyb & 6-31+G* & 0.0177 & 0.0001 & 0.2938 \\
TD-CAM-B3LYP & aug-cc-pVTZ & 0.0154 & 0.0005 & 0.2311 \\
SA(4)-CASSCF(12,11) & MB & 0.0005 & 0.0014 & 0.3832
\end{tabular}

${ }^{a}$ All data are from this work, calculated at the equilibrium geometry optimized by M06-2X/MG3S, unless specified otherwise. ${ }^{b}$ Ref. 14 .

(Table 5) suggest the same assignment as ref. 13 and 14 . Interestingly, our TD-B3LYP/MB calculation disagrees with the TD-B3LYP/6-31G(d) calculation in ref. 53 on the nature of the states while our calculation agrees with ref. 14 . The reason why the TD-B3LYP/6-31G(d) calculation in ref. 53 gave incorrect results is that the lack of diffuse functions in the $6-31 \mathrm{G}(\mathrm{d})$ basis set fails to properly describe the $n \sigma^{*}$ state because it has a significant amount of Rydberg character, which makes their calculated excitation energy for the first $n \sigma^{*}$ state (which should be $S_{2}$ ) higher than the second $\pi \pi^{*}$ state (which should be $S_{3}$ ). We confirmed this explanation of the source of error in their calculation by performing a TD-B3LYP/6-31+G(d) calculation, which gives the first $n \sigma^{*}$ state lower than the second $\pi \pi^{*}$ state.

To further clarify the nature of states and the interpretation of the electronic spectrum, we simulated the spectrum in the vapor phase using a normal-mode sampling approach as described in Section 2.3. The experimental spectrum shown in Fig. 3(a), as a reproduction of Fig. S6 in the Electronic Supplementary Information of ref. 14, has two closely located peaks at $239 \mathrm{~nm}(5.19 \mathrm{eV})$ and $250 \mathrm{~nm}(4.96 \mathrm{eV})$ respectively. We aim to simulate the lower-energy peak at $250 \mathrm{~nm}$ only, in order to verify our understanding of the lowest excited states (but we will discuss the other peak later in this section). We are interested in the three lowest excitations because we expect that, according to our calculated vertical excitations, the second $\pi \pi^{*}$ state $\left(S_{3}\right.$ at the equilibrium geometry) may be responsible for the strong band at $250 \mathrm{~nm}$. In practice we calculated the four lowest excitations because at some sampled geometries the second $\pi \pi^{*}$ state is $S_{4}$.

The simulated spectrum shown in Fig. 3(b) has a strong band peaked at $243 \mathrm{~nm}(5.10 \mathrm{eV})$ and a tail extending to longer wavelengths. Compared to Fig. 3(a), Fig. 3(b) has an overall shift to higher energy, but the profile reasonably resembles the experiment. Although the peak of the experimental spectrum is at $250 \mathrm{~nm}(4.96 \mathrm{eV}), 0.14 \mathrm{eV}$ is an acceptable error for this type of simulation. The other peak of Fig. 3(a) at higher energy does not appear in the simulation, which will be discussed further later. Despite the small quantitative differences, this simulation further supports our characterization of the excited states and our interpretation of the electronic spectrum.

Another important datum here is that the peak of the simulated spectrum $(5.10 \mathrm{eV})$ shifts to longer wavelength compared to the VEE calculated at the same TDA- $\tau$-HCTChyb/6-31+G* level
$(5.18 \mathrm{eV})$ by a non-negligible amount $(0.08 \mathrm{eV})$. This result is computed with harmonic vibrations, and if anharmonicity were taken into account the shift could be even larger. ${ }^{54}$ We also note that calculated transition moments can be hugely influenced by vibronic interaction, as illustrated in a recent theoretical study of the electronic absorption spectrum pyrrole. ${ }^{55}$ It is therefore worthwhile to state, although it is not a new observation, that one should not take the wavelength of the peak of an unresolved electronic spectrum as a "benchmark" of VEE, a conclusion that has long been recognized but is still often ignored.

A noteworthy point is that our TDDFT (with B3LYP and $\tau$-HCTHhyb) and SA-CASSCF calculations do not reveal another electronic state energetically near the bright $\pi \pi^{*}$ state that has comparable transition probability to that state, at either the equilibrium geometry or nearby geometries in the FranckCondon region. Accordingly the two peaks of the experimental spectrum at 239 and $250 \mathrm{~nm}$ may correspond to the same electronic state but be split vibronically, although the splitting is rather large $(0.23 \mathrm{eV})$. More study will be needed to clarify why there are two peaks. We emphasize that the aim of the present simulation is to clarify the nature of the excited states and the spectrum-not to quantitatively reproduce the experimental band shape. Achieving a quantitative simulation of the band shape, including reproducing the fine features at $\sim 280$ $290 \mathrm{~nm}$, may require more realistic sampling of the groundstate potential energy surface, explicitly taking into account the anharmonicity of the surfaces and hot bands, and accounting for the quantization of vibrations.

The transitions between the states can be further understood by examining their oscillator strengths and transition dipole moments. All of these transitions are allowed since in the $C_{\mathrm{s}}$ symmetry the $x$ and $y$ components of the dipole operator transform as the $\mathrm{A}^{\prime}$ irrep and the $z$ component as the $\mathrm{A}^{\prime \prime}$ irrep, and accordingly the $x$ and $y$ components of the dipole operator couple $\mathrm{S}_{1}$ and $\mathrm{S}_{3}\left(\mathrm{~A}^{\prime}\right)$ to $\mathrm{S}_{0}\left(\mathrm{~A}^{\prime}\right)$ and the $z$ component couples $\mathrm{S}_{2}\left(\mathrm{~A}^{\prime \prime}\right)$ to $\mathrm{S}_{0}$. As displayed in Table 5, SA-CASSCF, EOM-CCSD, and TDDFT qualitatively agree on the large oscillator strength of the $\mathrm{S}_{3} \leftarrow \mathrm{S}_{0}$ transition. EOM-CCSD and TDDFT agree as well on the oscillator strengths of $\mathrm{S}_{1} \leftarrow \mathrm{S}_{0}$ being one order of magnitude smaller than that of $\mathrm{S}_{3} \leftarrow \mathrm{S}_{0}$ and on the oscillator strength of $\mathrm{S}_{2} \leftarrow \mathrm{S}_{0}$ being negligible. The small oscillator strength of the $\mathrm{S}_{2} \leftarrow \mathrm{S}_{0}$ transition may be attributed to the relatively small spatial overlap of the $\mathrm{n}\left(p_{Z}\right)$ and $\sigma^{*}$ orbitals involved in the transition. SA-CASSCF predicts the oscillator strength of $S_{1} \leftarrow S_{0}$ to be smaller than of $S_{2} \leftarrow S_{0}$, both two orders of magnitudes smaller than that of $S_{3} \leftarrow S_{0}$, which is inaccurate due to lack of dynamical correlation.

\section{Concluding remarks}

In this paper, we clarified the nature of the four lowest singlet states of thioanisole at the equilibrium geometry using various wave function and density functional methods. We simulated the electronic absorption spectrum using a normal mode 
sampling approach, and its good agreement with experiment confirms our understanding of the spectrum, which is as follows. The transitions from $S_{0}$ (closed-shell) to $S_{1}\left(\pi \pi^{*}\right)$ and to $S_{2}\left(n \sigma^{*}\right)$ both have relatively small probability and contribute to the low-energy tail of the spectrum, whereas $S_{3} \leftarrow S_{0}$ is mainly responsible for the bright band at $250 \mathrm{~nm}$. We also confirmed that TDDFT, CR-EOM-CCSD(T), MC-QDPT, and XMC-QDPT are all reasonably accurate for computing the excitation energies. For potential energy surfaces, however, MC-QDPT and XMC-QDPT are more desirable at the current stage of development. Since XMC-QDPT generates smoother potential energy surfaces near state intersections, ${ }^{36}$ it appears suitable for future work of constructing the potential energy surfaces of thioanisole.

\section{Acknowledgements}

The authors are grateful to Dr Ke R. Yang, Dr Jingjing Zheng, and Professor Piotr Piecuch for many helpful discussions. We also thank Professor Vasilios G. Stavros and Mr Jamie D. Young for providing us with the experimental data of the UV/Vis spectrum of thioanisole. This work was supported in part by the U. S. Department of Energy, Office of Basic Energy Sciences, under grant no. DE-SC0008666. S.L.L. was also supported by the Dr V. Pothapragada Excellence Fellowship in Chemistry at the University of Minnesota.

\section{References}

1 A. L. Sobolewski and W. Domcke, Chem. Phys. Lett., 1999, 315, 293-298.

2 A. L. Sobolewski and W. Domcke, Chem. Phys., 2000, 259, 181-191.

3 A. L. Sobolewski and W. Domcke, J. Phys. Chem. A, 2001, 105, 9275-9283.

4 A. L. Sobolewski, W. Domcke, C. Dedonder-Lardeux and C. Jouvet, Phys. Chem. Chem. Phys., 2002, 4, 1093-1100.

5 Z. Lan, W. Domcke, V. Vallet, A. L. Sobolewski and S. Mahapatra, J. Chem. Phys., 2005, 122, 224315.

6 M. G. D. Nix, A. L. Devine, B. Cronin, R. N. Dixon and M. N. R. Ashfold, J. Chem. Phys., 2006, 125, 133318.

7 O. P. J. Vieuxmaire, Z. Lan, A. L. Sobolewski and W. Domcke, J. Chem. Phys., 2008, 129, 224307.

$8 \mathrm{X} . \mathrm{Xu}, \mathrm{K} . \mathrm{R}$. Yang and D. G. Truhlar, J. Chem. Theory Comput., 2013, 9, 3612-3625.

9 X. Xu, J. Zheng, K. R. Yang and D. G. Truhlar, J. Am. Chem. Soc., 2014, 136, 16378-16386.

10 K. R. Yang, X. Xu, J. Zheng and D. G. Truhlar, Chem. Sci., 2014, 5, 4661-4680.

11 I. S. Lim, J. S. Lim, Y. S. Lee and S. K. Kim, J. Chem. Phys., 2007, 126, 034306.

12 R. Omidyan and H. Rezaei, Phys. Chem. Chem. Phys., 2014, 16, 11679-11689.

13 J. S. Lim and S. K. Kim, Nat. Chem., 2010, 2, 627-632.
14 G. M. Roberts, D. J. Hadden, L. T. Bergendahl, A. M. Wenge, S. J. Harris, T. N. V. Karsili, M. N. R. Ashfold, M. J. Paterson and V. G. Stavros, Chem. Sci., 2013, 4, 993-1001.

15 T. H. Dunning, J. Chem. Phys., 1989, 90, 1007-1023.

16 D. E. Woon and T. H. Dunning, J. Chem. Phys., 1993, 98, 1358-1371.

17 T. H. Dunning, K. A. Peterson and A. K. Wilson, J. Chem. Phys., 2001, 114, 9244-9253.

18 B. J. Lynch, Y. Zhao and D. G. Truhlar, J. Phys. Chem. A, 2003, 107, 1384-1388.

19 R. Krishnan, J. S. Binkley, R. Seeger and J. A. Pople, J. Chem. Phys., 1980, 72, 650-654.

20 T. Clark, J. Chandrasekhar, G. W. Spitznagel and P. V. R. Schleyer, J. Comput. Chem., 1983, 4, 294-301.

21 E. Papajak, J. Zheng, X. Xu, H. R. Leverentz and D. G. Truhlar, J. Chem. Theory Comput., 2011, 7, 3027-3034.

22 W. J. Hehre, R. Ditchfield and J. A. Pople, J. Chem. Phys., 1972, 56, 2257-2261.

23 P. C. Hariharan and J. A. Pople, Theor. Chim. Acta, 1973, 28, 213-222.

24 M. M. Francl, W. J. Pietro, W. J. Hehre, J. S. Binkley, M. S. Gordon, D. J. DeFrees and J. A. Pople, J. Chem. Phys., 1982, 77, 3654-3665.

25 Y. Zhao and D. G. Truhlar, Theor. Chem. Acc., 2008, 120, 215-241.

26 M. E. Casida, in Recent Advances in Density Functional Methods, Part I, ed. D. P. Chong, World Scientific, Singapore, 1995, pp. 155-192.

27 (a) A. D. Becke, Phys. Rev. A: At., Mol., Opt. Phys., 1988, 38, 3098-3100; (b) C. T. Lee, W. T. Yang and R. G. Parr, Phys. Rev. B: Condens. Matter Mater. Phys., 1988, 37, 785-789; (c) B. Miehlich, A. Savin, H. Stoll and H. Preuss, Chem. Phys. Lett., 1989, 157, 200-206; (d) A. D. Becke, J. Chem. Phys., 1993, 98, 5648-5652.

28 I. Tamm, J. Phys., 1945, 9, 449-460.

29 S. M. Dancoff, Phys. Rev., 1950, 78, 382-385.

30 S. Hirata and M. Head-Gordon, Chem. Phys. Lett., 1999, 314, 291-299.

31 A. D. Boese and N. C. Handy, J. Chem. Phys., 2002, 116, 9559-9569.

32 M. W. Schmidt and M. S. Gordon, Annu. Rev. Phys. Chem., 1998, 49, 233-266.

33 H.-J. Werner and W. Meyer, J. Chem. Phys., 1981, 74, 5794-5801.

34 H. Nakano, J. Chem. Phys., 1993, 99, 7983.

35 H. Nakano, Chem. Phys. Lett., 1993, 207, 372-378.

36 A. A. Granovsky, J. Chem. Phys., 2011, 134, 214113.

37 H. Koch and P. Jørgensen, J. Chem. Phys., 1990, 93, 3333-3344.

38 J. F. Stanton and R. J. Bartlett, J. Chem. Phys., 1993, 98, 7029-7039.

39 K. Kowalski and P. Piecuch, J. Chem. Phys., 2004, 120, 1715-1738.

40 K. Kowalski, J. Chem. Phys., 2009, 130, 194110.

41 J. J. P. Stewart, J. Comput. Chem., 1989, 10, 209-220.

42 J. J. P. Stewart, J. Comput. Chem., 1989, 10, 221-264. 
43 M. Frisch, G. W. Trucks, H. B. Schlegel, G. E. Scuseria, M. A. Robb, J. R. Cheeseman, G. Scalmani, V. Barone, B. Mennucci, G. A. Petersson, H. Nakatsuji, M. Caricato, X. Li, H. P. Hratchian, A. F. Izmaylov, J. Bloino, G. Zheng, J. L. Sonnenberg, M. Hada, M. Ehara, K. Toyota, R. Fukuda, J. Hasegawa, M. Ishida, T. Nakajima, Y. Honda, O. Kitao, H. Nakai, T. Vreven, J. A. Montgomery, Jr., J. E. Peralta, F. Ogliaro, M. Bearpark, J. J. Heyd, E. Brothers, K. N. Kudin, V. N. Staroverov, R. Kobayashi, J. Normand, K. Raghavachari, A. Rendell, J. C. Burant, S. S. Iyengar, J. Tomasi, M. Cossi, N. Rega, J. M. Millam, M. Klene, J. E. Knox, J. B. Cross, V. Bakken, C. Adamo, J. Jaramillo, R. Gomperts, R. E. Stratmann, O. Yazyev, A. J. Austin, R. Cammi, C. Pomelli, J. W. Ochterski, R. L. Martin, K. Morokuma, V. G. Zakrzewski, G. A. Voth, P. Salvador, J. J. Dannenberg, S. Dapprich, A. D. Daniels, Ö. Farkas, J. B. Foresman, J. V. Ortiz, J. Cioslowski and D. J. Fox, Gaussian 09, Revision D.01, Gaussian, Inc., Wallingford CT, 2009.

44 M. W. Schmidt, K. K. Baldridge, J. A. Boatz, S. T. Elbert, M. S. Gordon, J. H. Jensen, S. Koseki, N. Matsunaga, K. A. Nguyen, S. J. Su, T. L. Windus, M. Dupuis and J. A. Montgomery, J. Comput. Chem., 1993, 14, 1347-1363.

45 M. S. Gordon and M. W. Schmidt, in Theory and Applications of Computational Chemistry: The First Forty Years, ed. C. E. Dykstra, G. Frenking, K. S. Kim and G. E. Scuseria, 2005, pp. 1167-1189.

46 M. Valiev, E. J. Bylaska, N. Govind, K. Kowalski, T. P. Straatsma, H. J. J. Van Dam, D. Wang, J. Nieplocha, E. Apra, T. L. Windus and W. de Jong, Comput. Phys. Commun., 2010, 181, 1477-1489.
47 J. Zheng, Z.-H. Li, A. W. Jasper, D. A. Bonhommeau, R. Valero, R. Meana-Pañeda and D. G. Truhlar, ANT, version 2014-2, University of Minnesota, Minneapolis, 2015, http:// comp.chem.umn.edu/ant.

48 J. J. P. Stewart, L. Fiedler, P. Zhang, J. Zheng, I. Rossi, W.-P. Hu, G. C. Lynch, Y.-P. Liu, Y.-Y. Chuang, J. Pu, J. Li, C. J. Cramer, P. L. Fast, J. Gao and D. G. Truhlar, MOPAC, version $5.021 \mathrm{mn}$, University of Minnesota, Minneapolis, MN, 55455, 2013.

49 T. Lu and F. Chen, J. Comput. Chem., 2012, 33, 580-592.

50 MG3S is a well-established basis set for ground-state geometry optimization with DFT, but to check for basis set effects we have also performed the optimization with another well-established basis set, namely the Turbomole basis set def2-TZVP, and the optimized coordinates differ from the ones with MG3S by only $\sim 0.001 \AA$.

51 M. Hoshino-Nagasaka, T. Suzuki, T. Ichimura, S. Kasahara, M. Baba and S. Kawauchi, Phys. Chem. Chem. Phys., 2010, 12, 13243-13247.

52 M. Nagasaka-Hoshino, T. Isozaki, T. Suzuki, T. Ichimura and S. Kawauchi, Chem. Phys. Lett., 2008, 457, 58-61.

53 M.-X. Liu, B.-B. Xie, M.-J. Li, Y.-Y. Zhao, K.-M. Pei, H.-G. Wang and X. Zheng, J. Raman Spectrosc., 2013, 44, 440-446.

54 B. Lasorne, J. Jornet-Somoza, H. D. Meyer, D. Lauvergnat, M. A. Robb and F. Gatti, Spectrochim. Acta, Part A, 2014, 119, 52-58.

55 G. Wu, S. P. Neville, O. Schalk, T. Sekikawa, M. N. R. Ashfold, G. A. Worth and A. Stolow, J. Chem. Phys., 2015, 142, 074302. 56 H. A. Witek, Y.-K. Choe, J. P. Finley and K. Hirao, J. Comput. Chem., 2002, 23, 957-965. 